\title{
Potentiel de séquestration du carbone des agroforêts à base de caféier robusta (Coffea canephora var. robusta) dans les bassins de production du Cameroun
}

\author{
Arlende Flore NGOMENI ${ }^{1 *}$, Emmanuel Lucien Nomo BIDZANGA ${ }^{1}$, \\ Marie Louise AVANA ${ }^{2}$, Martin Ngankam TCHAMBA ${ }^{2}$ et Cédric Djomo CHIMI ${ }^{3}$ \\ ${ }^{1}$ Institut de Recherche Agricole pour le Développement (IRAD), BP 2123 Yaoundé, Cameroun. \\ ${ }^{2}$ Faculté d'Agronomie et des Sciences Agricoles (FASA), Université de Dschang, BP 222 Dschang, Cameroun. \\ ${ }^{3}$ Institut de Recherche Agricole pour le Développement (IRAD), Yokadouma, BP 136 Yokadouma, Cameroun. \\ *Auteur correspondant ; E-mail : ngflore@yahoo.fr, Tél : (+237)699770469 ; (+237)677256110.
}

\author{
Received: 13-09-2021 Accepted: 15-12-2021 $\quad$ Published: 31-12-2021
}

\section{RESUME}

Une étude en vue d'évaluer le potentiel de séquestration du carbone des Agroforêts à base de Caféiers Robusta (ACR) a été menée dans quatre sites contrastés par leurs caractéristiques biophysiques et socioéconomiques, et appartenant à trois bassins de production du café au Cameroun. L'approche méthodologique a porté sur la collecte des données d'inventaires dans 120 ACR de différents âges (30 par site); lesquelles ont permis d'estimer le potentiel de séquestration du carbone de façon non-destructive en utilisant les équations allométriques. Le potentiel de séquestration du carbone évalués ont varié significativement entre les sites, de

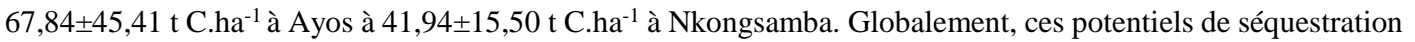
du carbone diminuaient suivant le gradient d'anthropisation des sites d'étude. Les ACR des classes d'âge (années) de [30-45[et de [45-60[ont eu les potentiels de séquestration du carbone les plus élevés pour les Espèces associées (EA) et les caféiers respectivement. Le potentiel de séquestration du carbone des EA endogènes $(19,46$ t C.ha $\left.{ }^{-1}\right)$ était plus élevé que celui des EA introduites $\left(6,32\right.$ t C.ha $\left.{ }^{-1}\right)$. Ces résultats montrent que la contribution des ACR dans les mécanismes d'atténuation des effets du changement climatique est une évidence qu'il faudrait capitaliser.

(C) 2021 International Formulae Group. All rights reserved.

Mots clés : Anthropisation, systèmes agroforestiers, zone agro écologique, Espèce associée.

\section{Carbon sequestration potential of robusta coffee agroforests (Coffea canephora var. robusta) in production basins of Cameroon}

\begin{abstract}
A study to assess the carbon sequestration potential of Robusta coffee Agroforests (RCAs) was carried out in four sites, contrasted by their biophysical and socio-economic characteristics, and belonging to three coffee production basins in Cameroon. The methodological approach involves the collection of inventory from 120
\end{abstract}


RCAs of different ages, (30 per site), which allowed no-destructive estimation of carbon stocks using. The assessed carbon sequestration potentials evaluated varied significantly between sites, from $67.84 \pm 45.41 \mathrm{Mg}$ C.ha ${ }^{-1}$ in Ayos to $41.94 \pm 15.50 \mathrm{Mg} \mathrm{C} \mathrm{ha}^{-1}$ in Nkongsamba. Overall, these carbon sequestration potentials decreased along the anthropization gradient of the study sites. RCAs of the [30-45[ and [45-60 [age classes (years) had the highest carbon sequestration potentials for associated species (Eas) and coffee trees respectively. The potential of carbon sequestration of the endogenous EA $\left(19.46 \mathrm{Mg} \mathrm{C}^{-h a^{-1}}\right)$ was higher than that of the

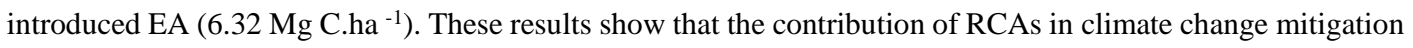
mechanisms is evident and should be capitalized upon.

(C) 2021 International Formulae Group. All rights reserved.

Keywords: Anthropisation, agroforestry systems, agro-ecological zone, associated species.

\section{INTRODUCTION}

Les écosystèmes en général fournissent aux êtres humains des biens, des services écologiques et socio-économiques nécessaires à leur bien-être et à leur développement (Ouattara et al., 2016). L'agroforesterie est un système de production qui permet une meilleure conservation de ces écosystèmes, et par conséquent, un pourvoyeur majeur des Services Ecosystémiques (SE) (Gomez et al., 2011). Les fonctions écosystémiques des Systèmes Agroforestiers (SAF) devraient être valorisées pour soutenir et protéger les activités humaines de production, de consommation et de protection de l'environnement (Power, 2010).

Les SAF à base de caféiers contiennent plusieurs Espèces Associées (EA) d'intérêts socio-économiques et écologiques (Fouellefack, 2015 ; Temgoua et al., 2020). Le choix de conserver ou d'introduire une espèce dans les SAF dépend de l'environnement biophysique et socio-économique du producteur. $\mathrm{La}$ séquestration naturelle $\mathrm{du}$ carbone est devenue de nos jours un enjeu important pour la stabilité du climat. L'approbation du mécanisme Réduction des Emissions dues à la Déforestation et la Dégradation des forêts (REDD) à Bali en 2007 a entraîné une mobilisation générale sur la nécessité de générer des informations sur les stocks de carbone dans les potentiels puits de carbone existants, y compris les systèmes d'utilisation des terres. Or, avec la dégradation des écosystèmes naturels en d'autres types d'utilisation des terres, il est évident que le potentiel de séquestration du carbone varie en fonction des pratiques agroforestières. Ainsi, les travaux de Albrecht et Kandji (2003) ont montré que le potentiel de séquestration du carbone est beaucoup plus élevé dans les SAF que dans les monocultures qui sont moins promotrices pour la conservation de la biodiversité. Ces auteurs ont trouvé des potentiels de séquestration du carbone qui variaient de 12 à $228 \mathrm{t} \mathrm{C.ha-1} \mathrm{dans} \mathrm{les} \mathrm{SAF} \mathrm{des}$ zones tropicales; et, la forte variabilité pourrait être fonction des EA, des régions phytogéographiques (climat, sol, etc.), des densités des espèces dans les plantations, de l'âge, de la gestion du système, etc. (Montagnini et Nair, 2004).

Au Cameroun, la plupart des travaux sur le potentiel de séquestration du carbone ont porté sur les agroforêts à base de cacaoyers (Zapfack et al., 2016; Silatsa et al., 2017; Madountsap et al., 2019). Les études portant sur les agroforêts à base de caféiers en général, et de caféiers robusta en particulier sont fragmentaires (Fouellefack, 2015 ; Temgoua et al., 2020), et ce en dépit d'importantes surfaces qu'occupe cette culture dans le paysage agraire du Cameroun. Il est ressorti de ces études, un potentiel de séquestration du carbone moyen variant de 24 à $43 \mathrm{t} \mathrm{C} \cdot$ ha $^{-1}$ dans les SAF à caféiers robusta. Cependant, les travaux menés à l'échelle mondiale par Avila (2002), Suarez (2002), Kurniatun (2002), Tadesse et al. (2014), Goodall et al. (2015), et Denu et al. (2016) ont montré une variation de l'ordre 
de 24 à 195 t C.ha-1 du potentiel de séquestration du carbone dans les SAF à caféiers robusta. Cette étude, qui visait à combler ce gap, a été menée dans trois bassins de production du caféier robusta au Cameroun. Elle avait pour objectif d'évaluer le potentiel de séquestration du carbone dans quatre sites contrastés par leur environnement écologique et socioéconomique.

\section{MATERIEL ET METHODES Sites d'étude}

Cette étude a été réalisée dans quatre sites de trois grands bassins de production du café robusta au Cameroun à savoir: (i) Nkongsamba et (ii) Mélong en zone de forêt dense humide à pluviométrie monomodale, à forte densité de population (355 et 205 $\mathrm{hbts} / \mathrm{Km}^{2}$ ) majoritairement migrante; Malantouen dans les hautes terres de l'Ouest à pluviométrie monomodale, à densité de population relativement faible $\left(42 \mathrm{hbts} / \mathrm{Km}^{2}\right)$ majoritairement autochtone; et, Ayos en zone de forêt dense humide à pluviométrie bimodale, à faible densité de population (18 $\left.\mathrm{hbts} / \mathrm{Km}^{2}\right)$ majoritairement autochtone. Ces trois bassins de production sont contrastés par leurs différentes caractéristiques biophysiques (Tableau 1) et conditions socio-économiques. Sur le plan phytogéographique (Onana, 2018 ; Onana et al., 2019), le bassin de production du Moungo appartient à la forêt atlantique biafréenne de basse et moyenne altitude, avec une végétation essentiellement constituée de galeries forestières très anthropisées à Nkongsamba, et dominée par les savanes à Mélong. Le bassin de production du CentreSud-Est appartient à la forêt dense humide semi-caducifoliée guinéo-congolaise de basse et moyenne altitude. Le bassin de production des Hauts Plateaux de l'Ouest est constitué des savanes arbustives et des galeries forestières. Les activités agricoles représentent les principales activités exercées dans ces zones, avec un accent sur les cultures de rente tel que le café.

\section{Méthodes}

\section{Dispositif d'échantillonnage}

Le dispositif des parcelles d'échantillonnages recommandé par Hairiah et al. (2010) a été utilisé dans le cadre de cette étude. Il repose sur la délimitation d'une unité d'échantillonnage rectangulaire d'un hectare au sein de chaque ACR à échantillonner. Cette forme tend à mieux inclure en son sein l'hétérogénéité spécifique des EA de la parcelle et par conséquent, est plus représentative que celle de forme carrée ou circulaire. Dans le cas de cette étude, compte tenu de la complexité de la structure des ACR, de l'irrégularité notée au niveau de leurs formes et de leurs tailles, des unités d'échantillonnages quelconques d'un hectare, qui représentaient au mieux la structure de la biodiversité végétale des ACR sélectionnées, ont été adoptées. Ainsi, un total de 120 ACR ont été échantillonnées soit, 30 par site d'étude.

\section{Collecte des données}

Le potentiel de séquestration du carbone de l'arbre a été évalué à partir de la biomasse totale de l'arbre, constituée des biomasses épigée et racinaire (GIEC, 2006). Dans chaque parcelle d'échantillonnage, outre les âges des ACR (suivant les classes d'amplitude de15 ans), les inventaires de toutes les EA de diamètre supérieur ou égal à $5 \mathrm{~cm}$ et tous les caféiers présents ont été collectés. Le diamètre a été mesuré à 1,3 $\mathrm{m}$ au-dessus du sol pour les EA et à $0,3 \mathrm{~m}$ au-dessus du sol pour les caféiers (Fouellefack, 2015). Les données d'abondance de chaque EA identifiée et leurs noms scientifiques/communs/vernaculaires ont aussi été collectés. La confirmation de leur identité scientifique a été faite à l'aide des lexiques botaniques ; il en était de même pour leur caractère endogène/conservatoire et introduite/exotique. Les données d'inventaires des EA et des caféiers ont été utilisées pour l'estimation de leur biomasse épigée et racinaire par la méthode non destructive. 


\section{Evaluation du potentiel de séquestration du carbone}

Le potentiel de séquestration $\mathrm{du}$ carbone a été évalué sur la base de la biomasse épigée, par la méthode non destructive à travers les équations allométriques de Chave et al. (2014), de Nyombi et al. (2009) et de Cummings et al. (2002), respectivement pour les ligneux (y compris caféiers), les palmiers et les Musaceae. Les formules sont définies comme suit :

$\mathrm{Y}_{\mathrm{e}}=\mathrm{e}^{(-1,803-0,976 \times \mathrm{E}+0,976 \ln (\rho)+2,673 \times \ln (\mathrm{D})-}$ $\left.0,0299 \times(\ln (\mathrm{D}))^{2}\right)$ (Chave et al., 2014) avec :

$\mathrm{Y}_{\mathrm{e}}=$ Biomasse aérienne $; \mathrm{E}=$ Indice environnemental $; \mathrm{D}=$ diamètre $; \rho=$ densité du bois.

Les densités spécifiques des espèces ont été extraites du «Global wood density data base » élaboré par Zanne et al. (2009).

$\mathrm{Y}=4,5+7,7 \times$ Hauteur (Cummings et al. 2002); avec $\mathrm{H}$ la hauteur des palmiers.

$\mathrm{Y}=0,325 \times \boldsymbol{e}^{0,036 \times \text { Circonférence (Nyombi et }}$ al. (2009); la circonférence représente celle des bananiers.
La biomasse racinaire a été obtenue en multipliant la valeur de la biomasse aérienne (AGB) par un coefficient R $(0,24)$.

Les potentiels de séquestration du carbone ont été déterminés en multipliant la somme des biomasses (épigée et racinaire) par le ratio Carbone Fraction (CF) de 0,47 (GIEC, 2006). Ainsi, la somme des potentiels de séquestration du carbone épigé de toutes les EA (EA ligneuses, caféiers, Musaceae et palmiers), a permis d'obtenir les potentiels de séquestration du carbone épigés totaux des ACR.

\section{Analyse statistique}

Le logiciel R (version 3.4.1) a été utilisé pour l'analyse des données. Le test de ShapiroWilk ayant montré que les données suivaient une loi normale, les tests paramétriques d'ANOVA et de Turkey's ont été utilisés pour voir si il existait une différence significative entre les quatre sites d'étude.

Tableau 1: Caractéristiques biophysiques des sites.

\begin{tabular}{|c|c|c|c|c|c|c|c|}
\hline $\begin{array}{l}\text { Zone agro } \\
\text { écologique }\end{array}$ & Site & $\begin{array}{l}\text { Coordonnées } \\
\text { géographiques }\end{array}$ & $\begin{array}{c}\text { Type de } \\
\text { climat }\end{array}$ & $\begin{array}{c}\text { Précipitatio } \\
\text { ns annuelles } \\
(\mathbf{m m})\end{array}$ & $\begin{array}{c}\text { Température } \\
\text { annuelle } \\
\text { moyenne }\end{array}$ & $\begin{array}{l}\text { Altitude } \\
\text { (m.a.s.l.) }\end{array}$ & Type de sol \\
\hline $\begin{array}{l}\text { Forêt dense } \\
\text { humide à } \\
\text { pluviométrie } \\
\text { mono modale }\end{array}$ & $\begin{array}{c}\text { Mélong } \\
\text { Nkongsamba }\end{array}$ & $\begin{array}{l}2^{\circ} 6 "-6^{\circ} 12^{\prime \prime} \mathrm{N} \\
8^{\circ} 48^{\prime \prime}-10^{\circ} 30^{\prime \prime} \mathrm{E}\end{array}$ & $\begin{array}{c}\text { Sub- } \\
\text { équatorial } \\
\text { avec } 2 \\
\text { saisons }\end{array}$ & $2500-4000$ & $26^{\circ} \mathrm{C}(2.8)$ & $400-780$ & $\begin{array}{l}\text { Ferralitiques, } \\
\text { volcaniques et } \\
\text { hydromorphes }\end{array}$ \\
\hline $\begin{array}{l}\text { Forêt dense } \\
\text { humide à } \\
\text { pluviométrie } \\
\text { bimodale }\end{array}$ & Ayos & $\begin{array}{l}2^{\circ} 6 "-4^{\circ} 54 " \mathrm{~N} \\
10^{\circ} 30^{\prime \prime}-16^{\circ} 12^{\prime \prime} \\
\mathrm{E}\end{array}$ & $\begin{array}{l}\text { Equatorial } \\
\text { guinéen } \\
\text { avec } 4 \\
\text { saisons }\end{array}$ & $1500-2000$ & $25^{\circ} \mathrm{C}(2.4)$ & $600-800$ & $\begin{array}{l}\text { Ferralitiques et } \\
\text { hydromorphes }\end{array}$ \\
\hline $\begin{array}{l}\text { Hauts } \\
\text { plateaux de } \\
\text { l'Ouest }\end{array}$ & Malantouen & $\begin{array}{c}4^{\circ} 54 "-6^{\circ} 36^{\prime \prime} \mathrm{N} \\
9^{\circ} 18^{\prime \prime}-11^{\circ} 24^{\prime \prime} \\
\mathrm{E}\end{array}$ & $\begin{array}{l}\text { Tropical } \\
\text { Soudano- } \\
\text { Guinéen } \\
\text { avec } 2 \\
\text { saisons }\end{array}$ & $1800-2400$ & $21^{\circ} \mathrm{C}(2.2)$ & $\begin{array}{l}1250- \\
2740\end{array}$ & $\begin{array}{c}\text { Latéritique, } \\
\text { alluvial et } \\
\text { ferralitique }\end{array}$ \\
\hline
\end{tabular}




\section{RESULTATS}

\section{Potentiel de séquestration du carbone des ACR dans les sites d'étude}

Les ACR ont un fort potentiel de séquestration du carbone. En prenant ces potentiels de séquestration du carbone par compartiment, ceux des caféiers ont varié significativement entre les sites (ANOVA, $\mathrm{p}=4,2^{-06}$ ), et suivaient l'ordre décroissant : (i) 45,18 $\pm 17,31$ t C.ha ${ }^{-1}$ à Mélong, (ii)

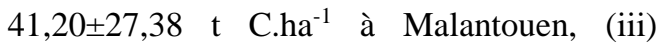
34,18 $\pm 13,46$ t C.ha ${ }^{-1}$ à Nkongsamba, et (iv)

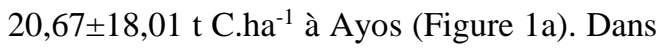
le cas des EA, ces potentiels de séquestration $\mathrm{du}$ carbone variaient aussi significativement entre les sites (ANOVA, $p=2,6^{-10}$ ), et suivaient

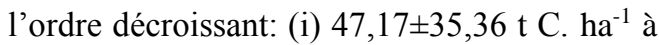
Ayos en zone de forêt humide; (ii) $22,82 \pm 18,44$ t C.ha ${ }^{-1}$ à Malantouen dans les hautes terres de l'ouest; (iii) 11,26 $\pm 11,40$ t C.ha $^{-1}$ et 7,59 $\pm 8,95$ $\mathrm{t} \mathrm{C}_{\mathrm{h}} \mathrm{h}^{-1}$ à Mélong et Nkongsamba respectivement en zone de forêt humide (Figure 1b).

Les potentiels de séquestration du carbone totaux (caféiers + EA) suivaient

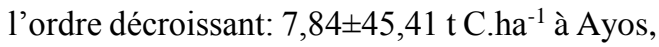
en zone de forêt humide à faible densité de population ; (ii) $64,01 \pm 29,02 \mathrm{t} \quad \mathrm{C} \cdot \mathrm{ha}^{-1}$ à Malantouen dans les hautes terres de l'ouest, moyennement peuplé ; et (iii) $56,43 \pm 22,96 \mathrm{t}$ C.ha ${ }^{-1}$ et $41,94 \pm 15,50$ t C.ha ${ }^{-1}$ à Mélong et Nkongsamba respectivement en zone de forêt humide à forte densité de population (Figure 1c), Ils étaient aussi significativement différents entre les sites (ANOVA, $\mathrm{P}=0$,00038). La contribution moyenne des EA au potentiel de séquestration du carbone total dans les ACR par site a été de : 69,53\% à Ayos, $35,65 \%$ à Malantouen, $19,95 \%$ à Mélong et $18,1 \%$ à Nkongsamba.

\section{Potentiel de séquestration du carbone en fonction des classes d'âge des ACR}

L'estimation du potentiel de séquestration du carbone moyen des ACR en fonction des classes d'âge des ACR étudiées, a montré qu'il variait d'une classe d'âge à l'autre. Dans le cas des caféiers, la tendance se rapprochait de celle d'une courbe en cloche renversée. Cette forme montre que le potentiel de séquestration du carbone augmente avec l'âge de l'ACR jusqu'à atteindre un pic (classe [45 - 60[ans), avant de décroitre pour les classes supérieures (Figure 2a). Par contre chez les EA, même si le pic est observé pour la classe [30-45[ans, la courbe semble ne pas se rapprocher de celle d'une loi normale (Figure $2 b)$.

\section{Potentiel de séquestration du carbone des} ACR en fonction des groupes taxonomiques

Des EA inventoriées, celles qui avaient les potentiels de séquestration du carbone les plus élevés en fonction des sites étaient: Albizia zygia $\left(6,45 \quad\right.$ t $\quad$ C.ha $\left.^{-1} ; \quad 28 \%\right)$ à Malantouen ; Musa paradisiaca (2,82 t C.ha ${ }^{-1}$; 25\%) à Mélong; Theobroma cacao (1,60 t C.ha $\left.^{-1} ; 21 \%\right)$ à Nkongsamba ; Pentaclethra macrophylla $\left(5,73\right.$ t C.ha $\left.^{-1} ; 12 \%\right)$ et Petersianthus macrocarpus (5,62 t C.ha ${ }^{-1}$; $12 \%$ ) à Ayos, ont eu le potentiel de séquestration du carbone le plus élevé dans chacun de ces sites. Les six espèces qui ont les potentiels de séquestration du carbone les plus élevés dans les ACR, sont essentiellement les EA introduites dans les sites de Mélong et de Nkongsamba, et les EA conservées dans les sites d'Ayos et de Malantouen (Tableau 2).

Des similitudes ont été observées entre les potentiels de séquestration du carbone des familles des EA dans les sites d'étude (Tableau 3). Les familles des Fabaceae et Malvaceae ont été communes à tous les sites d'étude comme faisant partie des familles à fort potentiel de séquestration du carbone. Cependant, les Fabaceae ont occupé le premier rang dans les sites de Malantouen, de Mélong et d'Ayos. Cette famille avait respectivement les potentiels de séquestration du carbone de 11,14 t C. ha $^{-1} ; 49 \% ; 14,61$ t C.ha ${ }^{-1} ; 31 \%$ et 3,66 t C.ha ${ }^{-1} ; 32 \%$ respectivement pour les sites de Ayos, Malantouen et Mélong, alors que dans le site de Nkongsamba, les Malvaceae ont

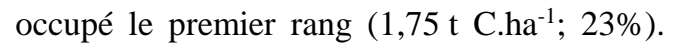
Les sites d'Ayos et de Malantouen avaient quatre familles en commun parmi les meilleures, ainsi que ceux de Nkongsamba et de Mélong. 


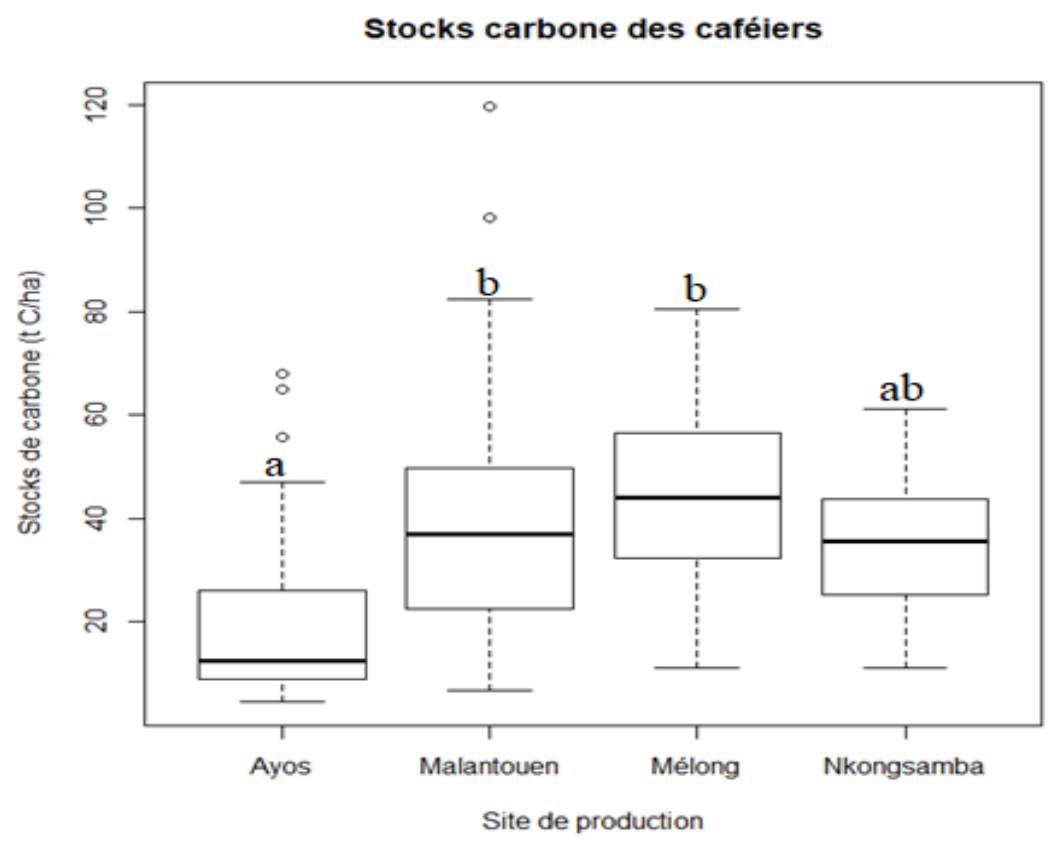

Figure 1a : Potentiel de séquestration du carbone des caféiers dans les agroforêts à base de caféiers robusta en fonction des sites.

\section{Stocks carbone des EA}

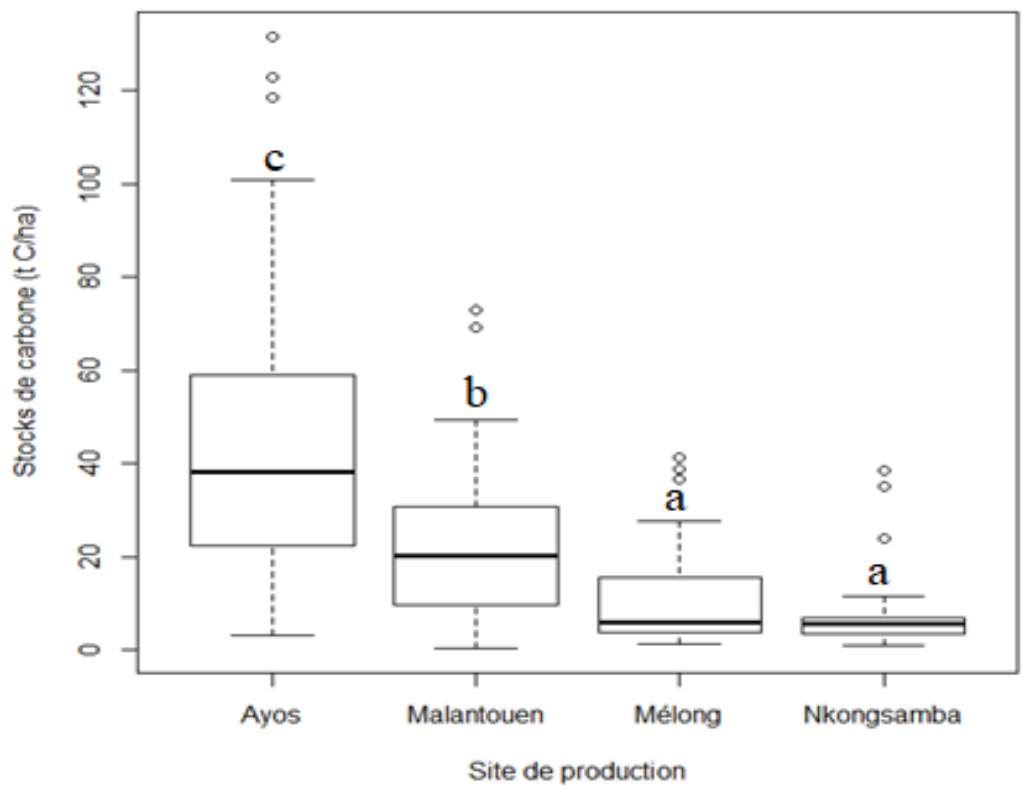

Figure 1b : Potentiel de séquestration du carbone des espèces associées en fonction des sites. 


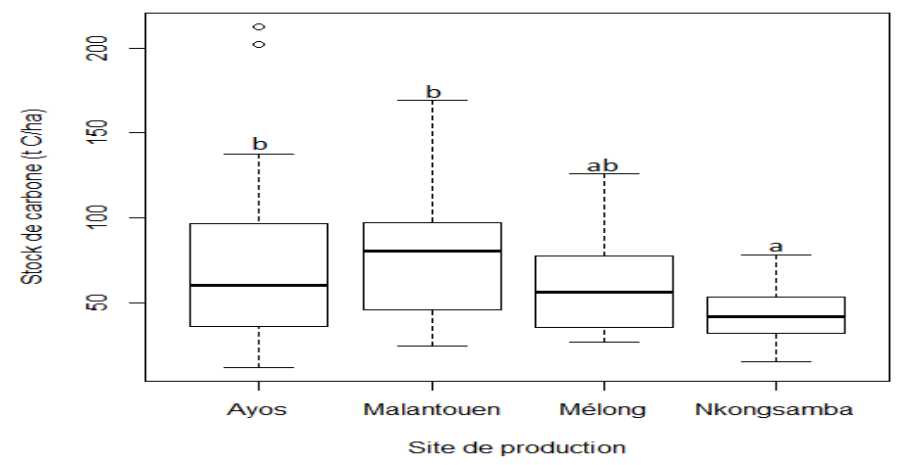

Figure 1c : Potentiel de séquestration du carbone total dans les agroforêts à base de caféiers robusta en fonction des sites.

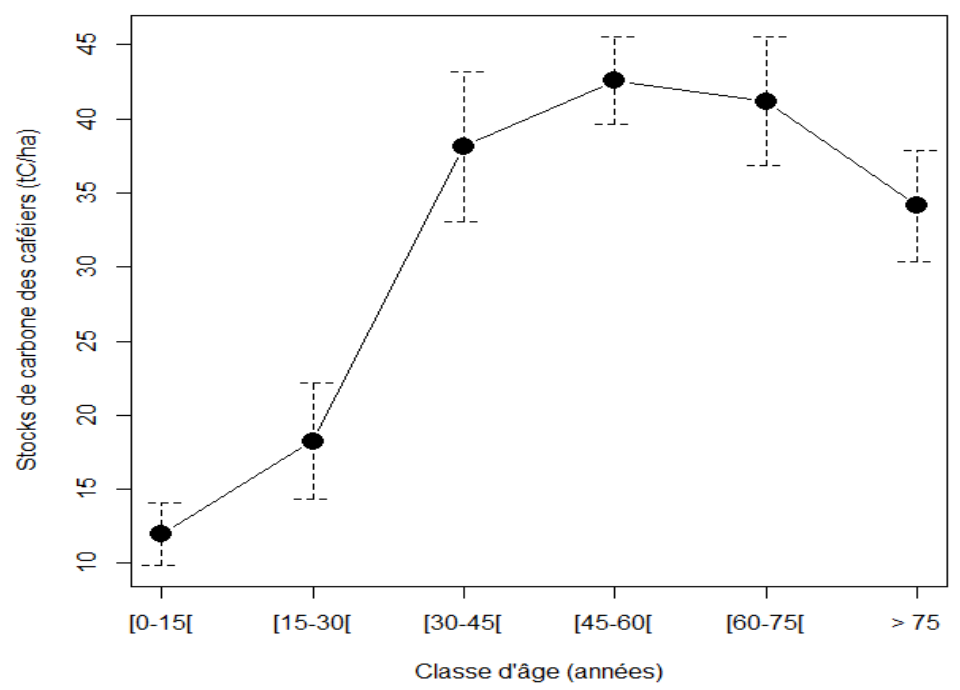

Figure 2a : Potentiel de séquestration du carbone des caféiers en fonction des classes d'âge des agroforêts à base de caféiers robusta.

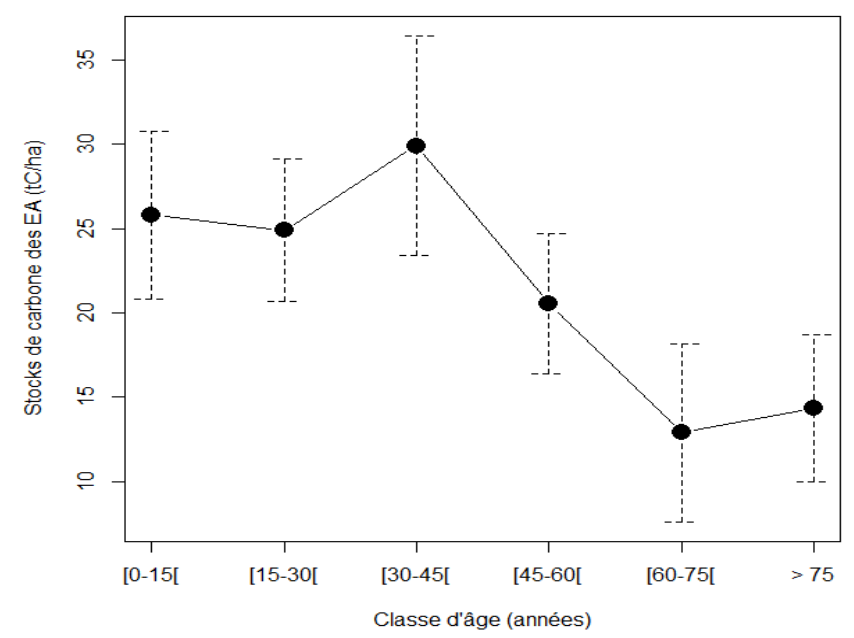

Figure 2b : Potentiel de séquestration du carbone des EA en fonction des classes d'âge des agroforêts à base de caféiers robusta. 
Tableau 2 : Espèces associées à potentiel de séquestration du carbone élevé dans les agroforêts à base de caféiers robusta par site.

\begin{tabular}{|c|c|c|c|c|}
\hline Rang & Nkongsamba & Mélong & Ayos & Malantouen \\
\hline 1 & $\begin{array}{l}\text { Theobroma cacao } \\
\left(1,60 \text { t C.ha }^{-1} ; 21 \%\right)\end{array}$ & $\begin{array}{l}\text { Musa paradisiaca } \\
\left(2,82 \text { t C.ha }^{-1} ; 25 \%\right)\end{array}$ & $\begin{array}{l}\text { Pentaclethra macrophylla } \\
\left(5,73 \mathrm{t} \mathrm{C} \mathrm{ha}^{-1} ; 12 \%\right)\end{array}$ & $\begin{array}{l}\text { Albizia zygia } \\
\left(6,45 \text { t C.ha }^{-1} ; 28 \%\right)\end{array}$ \\
\hline 2 & $\begin{array}{l}\text { Mangifera indica } \\
\left(1,31 \text { t C.ha }{ }^{-1} ; 17 \%\right)\end{array}$ & $\begin{array}{l}\text { Albizia glaberrima } \\
(2,43 \text { t C.ha-1 } ; 22 \%)\end{array}$ & $\begin{array}{l}\text { Petersianthus } \\
\text { macrocarpus }(5,62 \text { t C.ha- } \\
\text { 1; } 12 \%)\end{array}$ & $\begin{array}{l}\text { Milicia excelsa } \\
\left(3,91 \text { t C.ha }^{-1} ; 17 \%\right)\end{array}$ \\
\hline 3 & 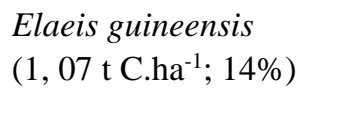 & 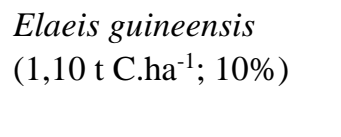 & $\begin{array}{l}\text { Albizia zygia } \\
\left(4,54 \text { t C.ha }{ }^{-1} ; 10 \%\right)\end{array}$ & $\begin{array}{l}\text { Piptadeniastrum } \\
\text { africanum (1, } 68 \text { t C.ha- }{ }^{-1} \text {; } \\
7 \%)\end{array}$ \\
\hline 4 & 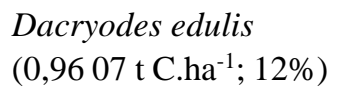 & $\begin{array}{l}\text { Dacryodes edulis } \\
(0,88 \mathrm{t} \mathrm{C/ha} ; 8 \%)\end{array}$ & $\begin{array}{l}\text { Ficus sp. } \\
(2,83 \text { t C.ha-1 } ; 6 \%) \text {, }\end{array}$ & $\begin{array}{l}\text { Albizia lebbeck } \\
\left(1,58 \text { t C.ha- }{ }^{-1} ; 7 \%\right)\end{array}$ \\
\hline 5 & $\begin{array}{l}\text { Erythrophleum } \\
\text { ivorense }\left(0,62 \text { t C.ha }^{-1} \text {; }\right. \\
11 \%)\end{array}$ & $\begin{array}{l}\text { Cola nitida } \\
\left(0,80 \text { t C.ha }^{-1} ; 7 \%\right)\end{array}$ & $\begin{array}{l}\text { Mangifera indica } \\
\left(2,45 \text { t C.ha }^{-1} ; 5 \%\right)\end{array}$ & $\begin{array}{l}\text { Ceiba pentandra } \\
\left(1,34 \text { t C.ha }^{-1} ; 6 \%\right)\end{array}$ \\
\hline 6 & $\begin{array}{l}\text { Persea americana } \\
\left(0,82 \text { t C.ha- }{ }^{-1} ; 11 \%\right)\end{array}$ & $\begin{array}{l}\text { Erythrophleum } \\
\text { ivorense }(0,74 \text { t C.ha-1 } \\
7 \%)\end{array}$ & $\begin{array}{l}\text { Bombax buonoposenze } \\
(2,39 \text { t C.ha-1; } 5 \%)\end{array}$ & $\begin{array}{l}\text { Albizia sp . } \\
(1,12 \text { t C.ha- } ; 5 \%)\end{array}$ \\
\hline
\end{tabular}

Tableau 3 : Familles à potentiel de séquestration du carbone élevé dans les agroforêts à base de caféiers robusta par site.

\begin{tabular}{|c|c|c|c|c|}
\hline Rang & Ayos & Malantouen & Nkongsamba & Mélong \\
\hline 1 & $\begin{array}{l}\text { Fabaceae } \\
\left(14,61 \text { t C} \text { ha }^{-1} ; 31 \%\right)\end{array}$ & $\begin{array}{l}\text { Fabaceae } \\
(11,14 \text { t C.ha-1 } ; 49 \%)\end{array}$ & $\begin{array}{l}\text { Malvaceae } \\
\left(1,75 \text { t C.ha }^{-1} ; 23 \%\right)\end{array}$ & $\begin{array}{l}\text { Fabaceae } \\
\left(3,66 \text { t C.ha }^{-1} ; 32 \%\right)\end{array}$ \\
\hline 2 & $\begin{array}{l}\text { Lecythidaceae } \\
\left(5,62 \mathrm{t} \mathrm{C}^{-1} \mathrm{ha}^{-1} ; 11,91 \%\right)\end{array}$ & $\begin{array}{l}\text { Moraceae } \\
\left(4,27 \text { t C.ha }{ }^{-1} ; 19 \%\right)\end{array}$ & $\begin{array}{l}\text { Anacardiaceae } \\
\left(1,31 \text { t C.ha }^{-1} ; 17 \%\right)\end{array}$ & $\begin{array}{l}\text { Musaceae } \\
\left(2,82 \text { t C. }^{-1} a^{-1} ; 25 \%\right)\end{array}$ \\
\hline 3 & $\begin{array}{l}\text { Moraceae } \\
\left(4,28 \text { t C.ha }{ }^{-1} ; 10 \%\right)\end{array}$ & $\begin{array}{l}\text { Bombacaceae } \\
\left(2,36 \text { t C.ha }^{-1} ; 10 \%\right)\end{array}$ & $\begin{array}{l}\text { Fabaceae } \\
\left(1,13 \text { t C.ha }{ }^{-1} ; 15 \%\right)\end{array}$ & $\begin{array}{l}\text { Malvaceae } \\
\left(1,17 \text { t C.ha }{ }^{-1} ; 10 \%\right)\end{array}$ \\
\hline 4 & $\begin{array}{l}\text { Bombacaceae } \\
\left(3,44 \text { t C.ha }^{-1} ; 7 \%\right)\end{array}$ & $\begin{array}{l}\text { Malvaceae } \\
\left(1,08 \text { t C.ha }^{-1} ; 5 \%\right)\end{array}$ & $\begin{array}{l}\text { Arecaceae } \\
\left(1,11 \text { t C.ha }{ }^{-1} ; 14 \%\right)\end{array}$ & $\begin{array}{l}\text { Arecaceae } \\
\left(1,11 \text { t C.ha }{ }^{-1} ; 10 \%\right)\end{array}$ \\
\hline 5 & $\begin{array}{l}\text { Malvaceae } \\
\left(3,24 \text { t C.ha }^{-1} ; 7 \%\right)\end{array}$ & $\begin{array}{l}\text { Verbenaceae } \\
\left(1,06 \text { t C.ha }^{-1} ; 5 \%\right)\end{array}$ & $\begin{array}{l}\text { Burseraceae } \\
\left(0,96 \text { t C.ha }^{-1} ; 12 \%\right)\end{array}$ & $\begin{array}{l}\text { Burseraceae } \\
\left(0,88 \text { t C.ha }^{-1} ; 8 \%\right)\end{array}$ \\
\hline 6 & $\begin{array}{l}\text { Anacardiaceae } \\
\left(2,62 \text { t C.ha- }^{-1} ; 6 \%\right)\end{array}$ & & $\begin{array}{l}\text { Lauraceae } \\
\left(0.82 \text { t C.ha }^{-1} ; 11 \%\right)\end{array}$ & \\
\hline
\end{tabular}




\section{DISCUSSION}

Les résultats obtenus montrent que les potentiels de séquestration du carbone des ACR des sites d'étude sont décroissants suivant l'ordre : Ayos, Malantouen, Mélong et Nkongsamba. Ceci pourrait être lié au contexte biophysique et socio-économique de chacun de ces sites. En effet, le site d'Ayos est caractérisé par la variabilité des facteurs susceptibles d'influencer le potentiel de séquestration du carbone des SAFs, notamment l'âge, les modes de gestion des ACR ; il en est aussi de même de la composition spécifique, la structure comme l'ont souligné certains auteurs (Zapfack et al., 2016 ; Sambieni et al., 2018 ; Temgoua et al., 2020). Par contre, les sites de Mélong et de Nkongsamba, qui appartiennent pourtant à une ère écologique similaire ont des potentiels de séquestration du carbone inférieurs. Ceci pourrait être attribuable au niveau d'anthropisation de ces sites dû à une forte activité humaine. En effet dans ces deux sites à forte densité de population majoritairement migrante, les producteurs sont confrontés au problème du foncier et, de par leur culture, impactent l'environnement dans lequel ils se trouvent en exploitant au maximum le petit espace de terre à leur disposition. Ils abattent les espèces indigènes ligneuses pour introduire les espèces exotiques, et du fait de l'accès au marché, les fruitiers en particulier qui leur rapportent des revenus supplémentaires substantiels par rapport à la culture principale (Montagnini et Nair, 2004 ; Sonwa et al., 2007 ; Madountsap et al., 2019) qui est le caféier robusta. Ainsi, du fait de leur caractère de jeunes arbres, ces EA introduites généralement de faible diamètre par rapport aux EA indigènes, ont un potentiel de séquestration du carbone faible comme c'est le cas à Mélong et à Nkongsamba.

A Ayos par contre, la disponibilité des terres et la faible anthropisation du paysage agraire du fait d'une faible densité de la population, et majoritairement composée d'autochtones, prédisposés à la conservation des EA indigènes à fort potentiel de séquestration du carbone. De plus, les producteurs peu équipés, ont des difficultés pour abattre les arbres de gros diamètre. Ils se trouvent dans l'obligation de les conserver dans les ACR, adaptant ainsi leurs activités à l'environnement. Ceci justifie le fort potentiel de séquestration du carbone moyen dans ce site, compte tenu de la conservation des arbres de gros diamètre dans les ACR par les producteurs (Zapfack et al., 2016).

A Malantouen, la relative faible densité de la population majoritairement autochtone couplée à l'enclavement du site dominé par les galeries forestières, n'encouragent pas les producteurs à une introduction massive des EA fruitières dans les ACR. Aussi, les producteurs de ce site de par leur culture, conservent volontairement certaines espèces à usages multiples dans leurs ACR. Ceci justifie le potentiel de séquestration du carbone élevé dans ce site par rapport aux sites de Mélong et de Nkongsamba qui se trouvent pourtant en zone de forêt dense humide. De manière générale, les potentiels de séquestration du carbone moyens des ACR diminuent suivant le gradient croissant d'anthropisation de ces sites, et donc dans l'ordre Ayos, Malantouen, Mélong et Nkongsamba.

Une dynamique d'évolution du potentiel de séquestration du carbone est observée avec l'âge des ACR. L'âge est généralement attribuable à la période de mise en place de la plantation sans tenir compte de l'âge des arbres qui s'y trouvent. Ainsi, les EA conservées lors de la mise en place des ACR, constituent un atout en termes de potentiel de séquestration du carbone contrairement aux EA introduites (fruitières), qui sont généralement plantées lors de la mise en place des plantations ou après (Fouellefack, 2015). Dans ce cas, malgré leur faible diamètre, leur abondance pourrait influencer le potentiel de séquestration du carbone. Ceci explique l'allure de la courbe 
des stocks de carbone des EA observée suivant les classes d'âge. En ce qui concerne les caféiers, une courbe se rapprochant de celle d'une cloche renversée a été observée pour les stocks de carbone. Ceci s'explique par le fait que, le potentiel de séquestration du carbone des caféiers augmente avec l'âge (compte tenu de l'augmentation de leur diamètre) jusqu'à atteindre un pilier qui caractérise leur maturité maximale. Par la suite, du fait du dépérissement, vieillissement et mort de certains caféiers (dans le cas des ACR très âgés), leur potentiel de séquestration du carbone commence à diminuer.

La structure des EA et des familles qui ont un potentiel de séquestration du carbone élevé dans les sites d'étude montre des similitudes et des dissemblances liées aux EA, à la gestion du système et à la zone agro écologique entre les sites, suggérant que le potentiel de séquestration du carbone dans les ACR dépend de la densité du bois des EA, de la gestion du système et de la zone agro écologique comme l'ont montré les travaux de Montagnini et Nair (2004).

Se limitant aux bassins de productions étudiés, les SAF qui prédominent dans ces zones sont essentiellement à base de caféiers et de cacaoyers; lesquels ont pratiquement les mêmes tendances managériales par les producteurs (Sonwa et al., 2007 ; Temgoua et al., 2020). Ainsi, Albrecht et Kandji (2003) pour les SAF tropicaux, montraient que dans les SAF, les potentiels de séquestration du carbone varient généralement de 12 à $228 \mathrm{t}$ C.ha ${ }^{-1}$ et, plusieurs facteurs pouvaient expliquer cette forte variabilité. Il s'agit notamment du type de SAF, de l'âge, des méthodes de gestion, la diversité, l'abondance et les classes de diamètre des EA, les conditions environnementales et la pédologie (Albrecht et Kandji ; 2003, Montagnini et Nair, 2004 ; Temgoua et al., 2020 ; Zapfack et al., 2016). Ces observations se rapprochent des résultats trouvés dans le cadre de cette étude ; et où les potentiels de séquestration du carbone trouvés variaient de 41,94 à 67,45 t C.ha- ${ }^{-1}$. Toutefois, dans le cas du bassin de production $\mathrm{du}$ Moungo, les valeurs moyennes trouvées dans les sites de Nkongsamba et de Mélong sont supérieures à celles trouvées par Temgoua et al. (2020) dans la localité de Kékem (24,28 t C.ha ${ }^{-1}$ ), qui appartient aussi pourtant au bassin de production du Moungo. Ceci pourrait s'expliquer par la différence entre les dispositifs d'échantillonnages utilisés, des méthodes de gestion appliquées par les producteurs de Kékem et aussi par le fait que Temgoua et al. (2020) par exemple n'ont pas pris en compte les bananiers présents dans les ACR lors de l'estimation du potentiel de séquestration du carbone. Dans le cas du bassin de production des hauts plateaux de l'Ouest, la valeur du potentiel de séquestration du carbone trouvée à Malantouen était proche des 37,143,2 t C.ha-1 trouvées par Fouellefack (2015) dans le même bassin de production.

\section{Conclusion}

Cette étude montre que les ACR des sites d'étude sont des potentiels puits de séquestration du carbone avec des quantités supérieures à $50 \mathrm{t} \mathrm{C} \cdot \mathrm{ha}^{-1}$. Elle révèle également l'influence des circonstances biophysiques et socio-économique, donc du mode de gestion des ACR sur le potentiel de séquestration du carbone de ceux-ci. La contribution moyenne des EA au potentiel de séquestration du carbone est généralement plus importante dans les sites peu anthropisés. Toutefois, le potentiel de ces EA varie en fonction des groupes taxonomiques et aussi d'un site à l'autre. Cette étude est une contribution remarquable à la promotion de la vente du potentiel de séquestration du carbone des $\mathrm{ACR}$, qui devrait être effective pour ces ACR au Cameroun, dans le but d'encourager les producteurs à une diversification des EA essentiellement endogènes à fort potentiel de séquestration du carbone. 


\section{CONFLITS D'INTERETS}

Les auteurs déclarent qu'ils n'ont pas d'intérêts concurrents.

\section{CONTRIBUTIONS DES AUTEURS}

AFN : conceptualisation de l'étude, collecte des données, rédaction du manuscrit ; ELNB : conceptualisation de l'étude, supervision des activités de terrain, apport par orientation et amendement du manuscrit; MLA et MNT : conceptualisation de l'étude, amendement du manuscrit; CDC: Analyse des données, apport par orientation et amendement du manuscrit.

\section{REMERCIEMENTS}

Les auteurs remercient particulièrement Dr. WOIN Noé, Directeur Général de l'Institut de Recherche agricole pour le Développement (IRAD), pour les fonds mis à disposition pour la réalisation de cette étude. Ces remerciements s'étendent aux facilitateurs sur le terrain et aux autres membres de l'équipe de collecte de données. Nous pensons notamment à : Tafou Bernard, Djomeni Emmanuel, Essambil David, Ngwanga Thomas, Galagou Emmanuel, Gapiensi David; Ntoupka Mama, Njouokou Salifou, Bekwake Alfred, Tchouga Fabrice, Nké blanche, Magne Doriane, Siéwé Cédric, Essong Robert, Tchuimessom Moïse et Tiako Pauline.

\section{REFERENCES}

Albrecht A, Kandji ST. 2003. Carbon sequestration in tropical agroforestry systems. Agric. Ecosyst. Environ., 99(3): 15-27.

DOI:

https://doi.org/10.1016/S01678809(03)00138-5

Avila VG. 2002. Fijacion y Almacenamiento de Carbono en Sistemas de café Bajo Sombra, Café a Pleno Sol, Sistemas Silvopastoriles y Pasturas a Pleno Sol. Turrilba, Costa Rica: CATI thesis, Turrilba, p. 205.
Chave J, Réjou-Méchain $\mathrm{M}$, Búrquez $\mathrm{A}$, Chidumayo E, Colgan MS, Delitti WBC, Duque A, Eid T, Fearnside PM, Goodman RC, Henry M, Martínez-Yrízar A, Mugasha W. 2014. Improved allometric models to estimate the aboveground biomass of tropical trees. Global Change Biol., 20: 3177-3190. DOI: http://dx.doi.org/10.1111/gcb.12629

Cummings D, Boone, KJ, Perry D, Hughes R. 2002. Aboveground Biomass and Structure of Rainforests in the Southwestern Brazilian Amazon. For. Ecol. Manage., 163: 293-307.

Denu D, Platts PJ, Kelbessa E, Tadesse WG, Marchant R. 2016. The role of traditional coffee management in forest conservation and carbon storage in the Jimma Highlands, Ethiopia. Forests Trees and Livelihoods, 25(4): 226-238. DOI: https://doi.org/10.1080/14728028.2016.1 192004

Fouellefack MVC, 2015. Diversité et services écosystémiques des ligneux dans les systèmes agroforestiers à base de caféiers du Département du Noun, Ouest Cameroun, Mémoire Master, Université de Dschang, Cameroun, Dschang, P. 92.

GIEC, 2006. Ligne directrice pour les inventaires nationaux de gaz à effet de serre: agriculture, foresterie et autres affectations des terres. Rapport de l'Institut des stratégies environnementales mondiales, Japon, 118.

Gomez DF, Rouspard O, le Maire G, Taugourdeau S, Pérez A, van Oijen M, Vaast P, Rapidel B, Harmand JM, Voltz M, Bonnefond JM, Imbach P, Moussa R. 2011. Modelling the hydrological behaviour of a coffee agroforestry basin in Costa Rica. Hydrology and Earth System Sciences, 15: 369-392.

Goodall KE, Bacon CM, Mendez VE. 2015. Shade tree diversity, carbon 
sequestration, and epiphyte presence in coffee agroecosystem: A decade of smallholder management in San Ramon, Nircanga. Agric. Ecosyst. Environ., 199: 200-206.

DOI: https://doi.org/10.1016/j.agee.2014.09.002

Hairiah K, Dewi S, Argus F, Velarde S, Ekadinata A, Rahayu S, Van Noordwijk M. 2010. Measuring Carbon Stocks Across Land Use Systems: A Manual. Bogor, Indonesia. World Agroforestry Centre (ICRAF), SEA Regional Office, 1-155.

Nyombi PJA, van Asten PA, Leffelaar M, Corbeels CK, Kaizzi KE Giller. 2009. Allometric growth relationships of East Africa highland bananas (Musa spp., AAA-EAHB) cv. Kisansa and Mbwazirume. Annals of Applied Biology, 155: 403-418.

Kurniatun H. 2002. Carbon stock assessment for a forest-to-coffee conversion landscape in Malang (East Java) and Sumber-Java (Lampung, Indonesia). In: Proceedings International Symposium on Forest Carbon and Monitoring, November 11-15, 2002 Taipei, Taivan, Tapei: Taiwan Forestry Research Institute. 28-36.

Madountsap TN, Zapfack L, Chimi DC, Kabelong Banoho LP, Forbi Preasios F, Tsopmejio TI, Tajeukem VC, Ntonmen YAF, Tabue MRB, Nasang JM. 2019. Carbon storage potential of cacao agroforestry systems of different age and management intensity. Climate and Development, 11(7): 543-554. DOI: https://doi.org/10.1080/17565529.2018.1 456895

Montagnini F, Nair PKR. 2004. Carbon sequestration: An underexploited environmental benefit of agroforestry systems. Agroforest. Syst., 61: 281-295.

Onana JM. 2018. Cartographie des écosystèmes du Cameroun. Int. J. Biol.
Chem. Sci., 12(2): 940-957. DOI: https://dx.doi.org/10.4314/ijbcs.v12i2.25

Onana JM, Fobane JL, Biye EH, Tchatchouang NE, Mbolo AMM. 2019. Habitats naturels des écosystèmes du Cameroun. Int. J. Biol. Chem. Sci., 13(7): 3247-3265. DOI:

https://dx.doi.org/10.4314/ijbcs.v13i7.22

Ouattara D, Kouame D, Tiebre M-S, Kouadio YJ-C, N'guessan KE. 2016. Biodiversité végétale et valeur d'usage en zone soudanienne de la Côte d'Ivoire. Int. J. Biol. Chem. Sci., 10(3): 1122-1138. DOI: http://dx.doi.org/10.4314/ijbcs.v10i3.18

Power AG. 2010. Ecosystem services and agriculture: trade-offs and synergies. Philosophical Transactions of the Royal Society B: Biological Sciences, 365: 2959-2971.

Sambieni KR, Bilosso MA, Toyi MS, Sambieni E, Natta AK, Occhiuto R, Bogaert J. 2018. La biodiversité des parcelles habitées en zone périurbaine à Kinshasa: déterminants sociobiophysiques et représentations. Int. J. Biol. Chem. Sci., 12(3): 1164-1183. DOI: https://dx.doi.org/10.4314/ijbcs.v12i3.8

Silatsa FBI, Yemefack M, Ewane-Nonga N, Kamga A, Hanna R. 2017. Modeling carbon stock dynamics under fallow and cocoa agroforest systems in the shifting agricultural landscape of central Cameroon. Agroforest. Syst., 91: 9931006.

DOI: http://dx.doi.org/10.1007/s10457-0169973-4

Sonwa DJ, Nkonmeneck BA, Weise SF, Tchatat M, Adesina AA, Janssens MJJ. 2007. Diversity of plants in cocoa agroforest in the humid forest zone of Southern Cameroon. Biodivers Conserv., 16: 2385-2400 DOI : http://dx.doi.org/10.1007/s10531-0079187-1 
Suarez D. 2002. Contificacion y Valoracion economica del Servicio Ambietal Almacenamiento de carbon en Sistemas Agroforestales de Café en la Comarca Yassica Sur, Metagalpa, Nicarangua, Turrialba, Costa Rica: CATIE thesis, p. 213.

Tadesse G, Zavaleta E, Shennan C. 2014. Effects of land-use changes on woody species distribution and above-ground carbon storage of forest-coffee systems. Agric. Ecosyst. Environ., 197: 21-30. DOI:

http://dx.doi.org/10.1016/j.agee.2014.07. 008

Temgoua LF, Etchike DAB, Solefack MMC, Tumenta P, Nkwelle Junior. 2020. Woody species diversity conservation and carbon sequestration potential of coffee agroforestry systems in the Western Region of Cameroon. Journal of Horticulture and Forestry, 12(2): 35-48. DOI: http://dx.doi.org/10.5897/JHF2020.0627. Zanne AE, Lopez-Gonzalez G, Coomes, DA, Ili CJ, Jansen S, Lewis SL, Chave, J. 2009. Global wood density database. http://www.hdl.handle.net/10255/dryad.2 35

Zapfack L, Chimi DC, Noiha Noumi V, Zekeng JC, Meyan-ya Daghela GR, Tabue Mbobda RB. 2016. Correlation between Associated Trees, Cocoa Trees and Carbon Stocks Potential in Cocoa Agroforests of Southern Cameroon. Sustainability in Environment, 1(2): 7184. 